\title{
LAY-UP OPTIMALITY CONDITIONS FOR BUCKLING LEVEL MAXIMIZATION OF VAT (STEERED FIBER) COMPOSITE PLATES
}

\author{
Sergey Selyugin \\ AIRBUS Operations GmbH, Kreetslag 10, 21129 Hamburg, Germany \\ e-mail: sergey.selyugin@airbus.com
}

\begin{abstract}
In the present paper the flat composite plates in buckling are studied. The plates have a symmetric lay-up and loaded along their contour by the in-plane forces. The consideration employs the Kirchhoff hypothesis and the von Karman approach. The lay-up optimality conditions for a single-mode (lowest) buckling eigenvalue are derived using the proper variational principles and the variation calculus. Both the bending terms and the terms following from the redistribution of the $2 \mathrm{D}$ stresses over the plate are taken into account. The physical meaning of the optimality conditions is discussed.
\end{abstract}

Keywords: composite plate, buckling, lay-up, steered fibers, optimality

(C) Sergey Selyugin, 2019 


\section{INTRODUCTION}

Buckling analysis of composite plates attracts attention of the numerous researchers since 80-ies years of the last century. The account of the effect allows obtaining lighter design solutions and using the capacity of the material more efficiently.

The book of Turvey and Marshall (1995) seems to be the first one summarizing the stateof-the-art for buckling and postbuckling of the composite plates. The more modern reviews may be found in the papers of Ghiasi et al. $(2009,2010)$ and in the book of Falzon and Aliabadi (2008).

We also mention some monographs important to the considered matter.

Ohsaki and Ikeda (2007) consider mainly the numerical aspects of the design optimization under the eigenvalue constraints.

In the book of Kassapoglou (2010) the modern approaches to the buckling analysis of the composite plates are described. The approaches are used at the Universities to educate the engineers in structural mechanics.

The buckling analysis of the composite plates is performed, as a rule, numerically, using the Rayleigh-Ritz or similar kinematic approaches based on the kinematic variational principles (indicated, e.g., in Washizu 1982).

In several papers (e.g., Ijsselmuiden et al. 2010) it was noticed in the numerical analysis that there is an influence of the 2D stress distribution on the buckling eigenvalue.

The present paper considers the influence theoretically. The theoretical results for the VAT plate buckling optimization are absent now.

The present paper deals with the derivation and analysis of the lay-up optimality conditions for the buckling level maximization of the steered fiber composite plates.

Section 1 presents the short Introduction. 
Section 2 describes the main assumptions and the theoretical background.

In Section 3 we derive the first variation of the buckling eigenvalue.

Section 4 is devoted to the result discussion.

Section 5 presents the conclusions.

\section{THEORETICAL BACKGROUND}

In the present paper we consider the laminated composite plate with the symmetric layup. The plate thickness is $h$. The lay-up is composed of the orthotropic fiber-reinforced plies of the same thickness $h_{p l y}$ and various point-wise orientation angles. The total number of plies is even and equal to $2 K$, the generalization to the odd ply number is straightforward and is not considered in the present paper. Fig. 1 illustrates some notations used in the paper.

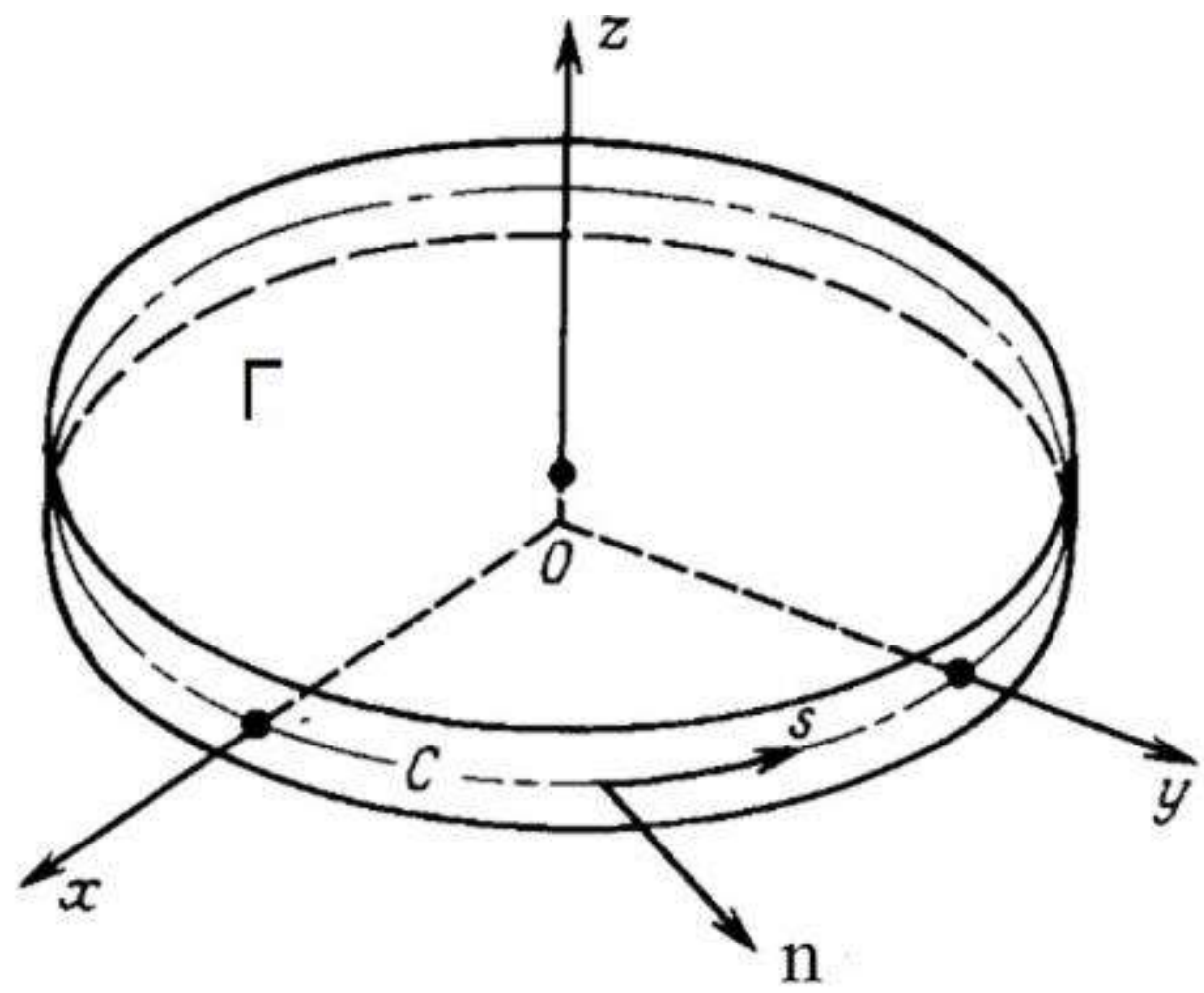

Fig. 1. 
The mid-plane $\Gamma$ of the flat plate is located in $X Y$ plane. The mid-surface is restricted by the piecewise smooth contour $C$ with the external normal $n$ (the components of the normal vector are $l, m)$ and the tangent direction $s$; the directions $n, s$ and $\mathrm{Z}$ create a right-hand triplet. The coordinate system $X Y Z$ is a Cartesian one.

The plate is loaded by the in-plane forces, acting at the part $C_{1}$ of the contour $C$. The remaining part $C_{2}$ of the contour the plate is not moving in $X$ and $Y$. The plate is simply supported in $\mathrm{Z}$ direction.

The $X, Y, Z$ displacements are denoted as $u, v, w$, respectively.

The signs of the loads are the following. The force flows $N_{x}, N_{y}$ (along $X$ and along $Y$ ) are positive in tension, the shear force flow $N_{x y}$ is positive when it decreases the $90^{\circ}$ angle between the orthogonal lines at the plate surface. The flows are in equilibrium. The Composite Lamination Plate Theory (CLPT) is used for the description of the plate deflections (see the book of Gibson 1994).

The Classical Lamination Plate Theory (CLPT), based on linear-elastic relations for every layer and Bernoulli's hypotheses, is used for obtaining the equation for the deflections $w$ (see the books of Gibson 1994, Lekhnitzky 1956, Reddy 2004). The equation is written as follows:

$$
\begin{aligned}
& D_{11} \frac{\partial^{4} w}{\partial x^{4}}+2\left(D_{12}+2 D_{66}\right) \frac{\partial^{4} w}{\partial x^{2} \partial y^{2}}+D_{22} \frac{\partial^{4} w}{\partial y^{4}}+4 D_{16} \frac{\partial^{4} w}{\partial x^{3} \partial y}+4 D_{26} \frac{\partial^{4} w}{\partial x \partial y^{3}}- \\
& -N_{x} \frac{\partial^{2} w}{\partial x^{2}}-N_{y} \frac{\partial^{2} w}{\partial y^{2}}-2 N_{x y} \frac{\partial^{2} w}{\partial x \partial y}=0
\end{aligned}
$$

where $D_{i j}, i=1,2,6 ; j=1,2,6$, are the elements of the bending stiffness matrix $D$, coupling the bending/twisting moments and various second derivatives of the deflection $w$ with respect to $x$ and $y$. The coupling is written as follows: 


$$
\left[\begin{array}{l}
M_{x} \\
M_{y} \\
M_{x y}
\end{array}\right]=\left[\begin{array}{lll}
D_{11} & D_{12} & D_{16} \\
D_{12} & D_{22} & D_{26} \\
D_{16} & D_{26} & D_{66}
\end{array}\right]\left[\begin{array}{c}
-\frac{\partial^{2} w}{\partial x^{2}} \\
-\frac{\partial^{2} w}{\partial y^{2}} \\
-2 \frac{\partial^{2} w}{\partial x \partial y}
\end{array}\right]
$$

We denote the left-hand side of (2) as a vector-column $\vec{M}$, and as $\vec{k}$ the vector-column at the right-hand side (which is multiplied to $D$ matrix). Also we use the notations

$$
k_{x}=-\frac{\partial^{2} w}{\partial x^{2}} ; k_{y}=-\frac{\partial^{2} w}{\partial y^{2}} ; k_{x y}=-\frac{\partial^{2} w}{\partial x \partial y}
$$

Then (2) is rewritten in the matrix-vector form as:

$$
\vec{M}=D \vec{k}
$$

Now we discuss the energy balance for the plate element $d x d y$. For a feasible deflection $\delta w$ we have the variation of the structural work of the internal forces (see the book of Volmir 1967)

$$
\delta A=-\iint d S\left(M_{x} \delta k_{x}+M_{y} \delta k_{y}+2 M_{x y} \delta k_{x y}\right)
$$

where $\delta$ is the variation symbol. Then the increase of the total strain potential energy $\widetilde{\Pi}$ is

$$
\delta \widetilde{\Pi}=-\delta A
$$

The quantity of the total strain potential energy and its variation are written as follows:

$$
\begin{aligned}
& \widetilde{\Pi}=\iint d S\left(\frac{1}{2} D_{11}\left(\frac{\partial^{2} w}{\partial x^{2}}\right)^{2}+D_{12} \frac{\partial^{2} w}{\partial x^{2}} \frac{\partial^{2} w}{\partial y^{2}}+\frac{1}{2} D_{22}\left(\frac{\partial^{2} w}{\partial y^{2}}\right)^{2}+\right. \\
& \left.+2 D_{16} \frac{\partial^{2} w}{\partial x^{2}} \frac{\partial^{2} w}{\partial x \partial y}+2 D_{26} \frac{\partial^{2} w}{\partial y^{2}} \frac{\partial^{2} w}{\partial x \partial y}+2 D_{66}\left(\frac{\partial^{2} w}{\partial x \partial y}\right)^{2}\right) \\
& \delta \widetilde{\Pi}=\delta \iint d S\left(\frac{1}{2} D_{11}\left(\frac{\partial^{2} w}{\partial x^{2}}\right)^{2}+D_{12} \frac{\partial^{2} w}{\partial x^{2}} \frac{\partial^{2} w}{\partial y^{2}}+\frac{1}{2} D_{22}\left(\frac{\partial^{2} w}{\partial y^{2}}\right)^{2}+\right. \\
& \left.+2 D_{16} \frac{\partial^{2} w}{\partial x^{2}} \frac{\partial^{2} w}{\partial x \partial y}+2 D_{26} \frac{\partial^{2} w}{\partial y^{2}} \frac{\partial^{2} w}{\partial x \partial y}+2 D_{66}\left(\frac{\partial^{2} w}{\partial x \partial y}\right)^{2}\right)
\end{aligned}
$$


The work, performed by the external forces, is

$$
W=-\frac{1}{2} \iint d S\left[N_{x}\left(\frac{\partial w}{\partial x}\right)^{2}+N_{y}\left(\frac{\partial w}{\partial y}\right)^{2}+2 N_{x y} \frac{\partial w}{\partial x} \frac{\partial w}{\partial y}\right]
$$

The total energy of the system

$$
U=\widetilde{\Pi}-W
$$

Because of energy balance at the buckling state $w$ the energy variation $\delta U$ is equal to zero:

$$
\delta U=\delta \widetilde{\Pi}-\delta W=0
$$

Making formal variations in (8)-(10), integrating by parts and taking into account the boundary conditions of the considered types and the in-plane equilibrium, we obtain the equilibrium equation (1). Hence, the stationarity conditions of (10) with respect to $w$, under the proper boundary conditions, lead to the buckling equation (1). The statement is a content of the kinematic variational principle for the plate buckling.

Following the book of Washizu (1982), the kinematic variational principle may be rewritten as the stationarity form of the following ratio (under the same boundary conditions as before):

$$
\delta\left[-\frac{\tilde{\Pi}}{W^{(0)}}\right]=0
$$

where

$$
W^{(0)}=-\iint d S\left[\frac{1}{2} N_{x}^{(0)}\left(\frac{\partial w}{\partial x}\right)^{2}+\frac{1}{2} N_{y}^{(0)}\left(\frac{\partial w}{\partial y}\right)^{2}+N_{x y}^{(0)} \frac{\partial w}{\partial x} \frac{\partial w}{\partial y}\right]
$$

and the superscript (0) corresponds to some constant initial (pre-buckling) force flows. The flows should be multiplied by the eigenvalue $\lambda$ for obtaining the buckling force flows.

For found buckling mode the eigenvalue satisfies the Rayleigh relation:

$$
\lambda=\frac{\widetilde{\Pi}}{W^{(0)}}
$$


The distribution of the in-plane stress flows are given by the relations:

$$
\vec{N}=A\left[\begin{array}{c}
u_{, x} \\
v_{, y} \\
u_{, y}+v_{, x}
\end{array}\right]
$$

where $A$ is the in-plane stiffness matrix. The rhs column-vector is

$$
\vec{N}=\left(N_{x}, N_{y}, N_{x y}\right)^{T}
$$

$T$ means transposing, and index after comma means the differentiation w.r.t. the corresponding coordinate.

The components of the $\vec{N}$ must satisfy the equilibrium equations, and the in-plane displacements must satisfy the corresponding boundary conditions.

We will also use below some relations for the laminated plate presented in Selyugin (2019a, 2019b).

For the orthotropic lamina, we follow the approach and the notations of (Gibson 1994, Section 2.6).

Fig. 2 shows the orthotropic lamina. The stress tensor components within the lamina in the local coordinates $(x, y)$ are:

$$
\left(\begin{array}{c}
\sigma_{x} \\
\sigma_{y} \\
\tau_{x y}
\end{array}\right)=\left[\begin{array}{lll}
\bar{Q}_{11} & \bar{Q}_{12} & \bar{Q}_{16} \\
\bar{Q}_{12} & \bar{Q}_{22} & \bar{Q}_{26} \\
\bar{Q}_{16} & \bar{Q}_{26} & \bar{Q}_{66}
\end{array}\right]\left(\begin{array}{c}
\varepsilon_{x} \\
\varepsilon_{y} \\
2 \varepsilon_{x y}
\end{array}\right)
$$

where $\bar{Q}$ is the lamina stiffness matrix, and $\varepsilon_{x}, \varepsilon_{y}, \varepsilon_{x y}$ are the lamina infinitesimal strain tensor components. 


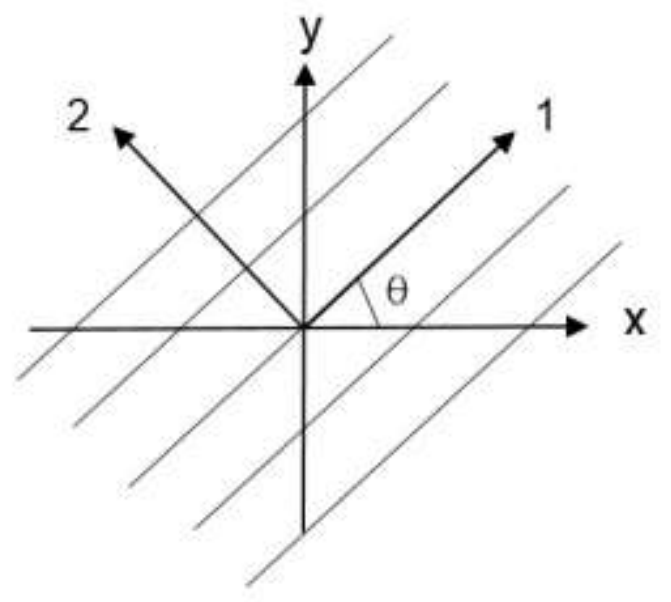

Fig. 2. Orthotropic lamina with material orientation $(1,2)$.

The components of the lamina stiffness matrix are (see Gibson 1994, Section 2.6):

$$
\begin{aligned}
& \bar{Q}_{11}=U_{1}+U_{2} \cos 2 \theta+U_{3} \cos 4 \theta \\
& \bar{Q}_{12}=U_{4}-U_{3} \cos 4 \theta \\
& \bar{Q}_{22}=U_{1}-U_{2} \cos 2 \theta+U_{3} \cos 4 \theta \\
& \bar{Q}_{16}=\frac{1}{2} U_{2} \sin 2 \theta+U_{3} \sin 4 \theta \\
& \bar{Q}_{26}=\frac{1}{2} U_{2} \sin 2 \theta-U_{3} \sin 4 \theta \\
& \bar{Q}_{66}=\frac{1}{2}\left(U_{1}-U_{4}\right)-U_{3} \cos 4 \theta
\end{aligned}
$$

where $U_{1}, U_{2}, U_{3}, U_{4}$ are the elastic constants of the orthotropic layer. The stress tensor components within the lamina in the coordinates $(x, y)$ are:

$$
\left(\begin{array}{c}
\sigma_{x} \\
\sigma_{y} \\
\tau_{x y}
\end{array}\right)=\left[\begin{array}{lll}
\bar{Q}_{11} & \bar{Q}_{12} & \bar{Q}_{16} \\
\bar{Q}_{12} & \bar{Q}_{22} & \bar{Q}_{26} \\
\bar{Q}_{16} & \bar{Q}_{26} & \bar{Q}_{66}
\end{array}\right]\left(\begin{array}{c}
\varepsilon_{x} \\
\varepsilon_{y} \\
2 \varepsilon_{x y}
\end{array}\right)
$$

where $\bar{Q}$ is the lamina stiffness matrix, and $\varepsilon_{x}, \varepsilon_{y}, \varepsilon_{x y}$ are the lamina infinitesimal strain tensor components. The components of the lamina stiffness matrix are the known algebraic 
functions of the four layer elastic constants $U_{1}, U_{2}, U_{3}, U_{4}$ and of the trigonometric functions of the angle $\theta$. The angle is the angle between the fiber direction and the $x$-axis (measured in the direction of the counter-clockwise rotation from $x$ to $y$ ).

According to (Gibson 1994), the derivatives of the lamina stiffness matrix $\bar{Q}_{i j}$ w.r.t. the orientation angle $\theta$ are:

$$
\begin{aligned}
& \frac{d \bar{Q}_{11}}{d \theta}=-2 U_{2} \sin 2 \theta-4 U_{3} \sin 4 \theta=-4 \bar{Q}_{16} \\
& \frac{d \bar{Q}_{22}}{d \theta}=2 U_{2} \sin 2 \theta-4 U_{3} \sin 4 \theta=4 \bar{Q}_{26} \\
& \frac{d \bar{Q}_{12}}{d \theta}=4 U_{3} \sin 4 \theta=2\left(\bar{Q}_{16}-\bar{Q}_{26}\right) \\
& \frac{d \bar{Q}_{16}}{d \theta}=U_{2} \cos 2 \theta+4 U_{3} \cos 4 \theta \\
& \frac{d \bar{Q}_{26}}{d \theta}=U_{2} \cos 2 \theta-4 U_{3} \cos 4 \theta \\
& \frac{d \bar{Q}_{66}}{d \theta}=4 U_{3} \sin 4 \theta
\end{aligned}
$$

The determination of $A_{i j}$ and $D_{i j}$ via the lamina parameters is performed according to the general formulas (Gibson 1994), $i j=11,12,22,16,26,66$ :

$$
\begin{aligned}
& A_{i j}=\sum_{k=1}^{K}\left(\bar{Q}_{i j}\right)_{k}\left(z_{k}-z_{k-1}\right) \\
& D_{i j}=\frac{1}{3} \sum_{k=1}^{K}\left(\bar{Q}_{i j}\right)_{k}\left(z_{k}^{3}-z_{k-1}^{3}\right)
\end{aligned}
$$

where $z_{k}, z_{k-1}$ are the top and the bottom $z$-coordinates of the layer ( $z=0$ corresponds to the mid-plane).

The derivatives of the stiffness matrices $A, D$ w.r.t. the lamina angles $\theta_{k}$ are, $i j=11,12$, $22,16,26,66 ; k=1, \ldots, K$ : 


$$
\begin{aligned}
& \frac{d A_{i j}}{d \theta_{k}}=\left(z_{k}-z_{k-1}\right) \frac{d\left(\bar{Q}_{i j}\right)_{k}}{d \theta_{k}} \\
& \frac{d D_{i j}}{d \theta_{k}}=\frac{1}{3}\left(z_{k}^{3}-z_{k-1}^{3}\right) \frac{d\left(\bar{Q}_{i j}\right)_{k}}{d \theta_{k}}
\end{aligned}
$$

where $\theta_{k}$ is the layer orientation angle.

\section{FIRST VARIATION OF THE BUCKLING EIGENVALUE}

Now we proceed with the determination of the variation of the eigenvalue. It is supposed that the eigenvalue is a single-modal one. Our goal is to design a lay-up maximizing the first (lowest) eigenvalue.

For making the variation we use the Lagrange function $L$ uniting the goal function and the constraint of the problem.

The considered constraints of the problem are:

- $\quad$ the equilibrium equations (Gibson, 1994)

$$
\begin{aligned}
& N_{x, x}^{(0)}+N_{x y, y}^{(0)}=0 \\
& N_{x y, x}^{(0)}+N_{y, y}^{(0)}=0
\end{aligned}
$$

- the loading conditions by the $\mathrm{X}$ and Y loads $\bar{N}_{x n}^{(0)}, \bar{N}_{y n}^{(0)}$ at $C_{1}$

$$
\begin{aligned}
& N_{x}^{(0)} l+N_{x y}^{(0)} m=\bar{N}_{x n}^{(0)} \\
& N_{x y}^{(0)} l+N_{y}^{(0)} m=\bar{N}_{y n}^{(0)}
\end{aligned}
$$

- the kinematic in-plane conditions at $C_{2}$

$$
\begin{aligned}
& u=\bar{u}=0 \\
& v=\bar{v}=0
\end{aligned}
$$

The subscript after comma above and below means the differentiation wrt to the subscript letter. 
The Lagrangian $L$ is written as follows:

$$
\begin{aligned}
& L=\frac{\widetilde{\Pi}}{W^{(0)}}+\int_{\Gamma} d \Gamma\left[\alpha_{1}\left(N_{x, x}^{(0)}+N_{x y, y}^{(0)}\right)+\alpha_{2}\left(N_{x y, x}^{(0)}+N_{y, y}^{(0)}\right)\right]+ \\
& +\int_{C_{1}} d C\left[\beta_{1}\left(N_{x}^{(0)} l+N_{x y}^{(0)} m-\bar{N}_{x n}^{(0)}\right)+\beta_{1}\left(N_{x y}^{(0)} l+N_{y}^{(0)} m-\bar{N}_{y n}^{(0)}\right)\right]+\int_{C_{2}} d C\left(\gamma_{1} u+\gamma_{2} v\right)+ \\
& +\int_{\Gamma} d \Gamma\left[\vec{\omega}^{T}\left(A^{-1} \vec{N}-\left[\begin{array}{c}
u_{, x} \\
v_{, y} \\
u_{, y}+v_{, x}
\end{array}\right]\right)\right]
\end{aligned}
$$

where $\alpha_{1}, \alpha_{2}, \beta_{1}, \beta_{2}, \gamma_{1}, \gamma_{2}, \omega_{1}, \omega_{2}, \omega_{3}$ are the Lagrange multipliers; the column-vector $\vec{\omega}$ is composed of $\omega_{1}, \omega_{2}, \omega_{3}$.

The Lagrangian variation (to be equal to zero for the optimal design) is written in the form:

$$
\begin{aligned}
& \delta L=\delta_{w}\left[\frac{\widetilde{\Pi}}{W^{(0)}}\right]+\delta_{D}\left[\frac{\widetilde{\Pi}}{W^{(0)}}\right]+\delta_{\vec{N}^{(0)}}\left[\frac{\widetilde{\Pi}}{W^{(0)}}\right]+\delta \int_{\Gamma} d \Gamma\left[\alpha_{1}\left(N_{x, x}^{(0)}+N_{x y, y}^{(0)}\right)+\alpha_{2}\left(N_{x y, x}^{(0)}+N_{y, y}^{(0)}\right)\right]+ \\
& +\delta \int_{C_{1}} d C\left[\beta_{1}\left(N_{x}^{(0)} l+N_{x y}^{(0)} m-\bar{N}_{x n}^{(0)}\right)+\beta_{1}\left(N_{x y}^{(0)} l+N_{y}^{(0)} m-\bar{N}_{y n}^{(0)}\right)\right]+\int_{C_{2}} d C\left(\gamma_{1} \delta u+\gamma_{2} \delta v\right)+ \\
& +\delta_{\vec{N}^{(0)}, u, v}\left[\int_{S} d S \vec{\omega}^{T}\left(A^{-1} \vec{N}^{(0)}-\left(u_{, x} ; v_{, y} ; u_{, y}+v_{, x}\right)^{T}\right)\right]+\sum_{i=1}^{K} \delta_{\theta_{i}} \int_{S} d S \vec{\omega}^{T} A^{-1} \vec{N}^{(0)}
\end{aligned}
$$

where the first item is equal to zero due to the above-indicated variational principle describing the plate stability, the second item is due to the bending stiffness variation and is derived in Selyugin (2013) (the influence of the in-plane force variation was considered as small and negligible there), the third item is due to the in-plane force variations influencing the eigenvalue, the fourth item corresponds to the in-plane equilibrium, the fifth item corresponds to the loading, the sixth item corresponds to the in-plane boundary conditions, the seventh item corresponds to (15), the eighth item corresponds to the ply orientation angle $\theta_{i}$, $i=1, \ldots, K$, variation influencing $(15)$.

Performing the usual transformations based on the Gauss divergence theorem, we obtain: 


$$
\begin{aligned}
& \delta_{\vec{N}^{(0)}}\left[\frac{\widetilde{\Pi}}{W^{(0)}}\right]=-\frac{1}{2} \frac{\lambda}{W^{(0)}} \int_{S} d S\left(w_{, x}^{2} \delta N_{x}+w_{, y}^{2} \delta N_{y}+2 w_{, x} w_{, y} \delta N_{x y}\right) \\
& \delta_{\vec{N}^{(0)}}\left[\int_{S} d S \vec{\omega}^{T}\left(A^{-1} \vec{N}-\left(u_{, x} ; v_{, y} ; u_{, y}+v_{, x}\right)^{T}\right)\right]=\int_{S} d S \vec{\omega}^{T} A^{-1} \delta \vec{N} \\
& \delta_{u, v}\left[\int_{S} d S \vec{\omega}^{T}\left(A^{-1} \vec{N}-\left(u_{, x} ; v_{, y} ; u_{, y}+v_{, x}\right)^{T}\right)\right]=\oint d l\left[\left(\omega_{1} \delta u+\omega_{3} \delta v\right) \cos (x, v)+\right. \\
& \left.+\left(\omega_{2} \delta v+\omega_{3} \delta u\right) \cos (y, v)\right]-\int_{S} d S\left[\delta u\left(\omega_{1, x}+\omega_{3, y}\right)+\delta v\left(\omega_{2, y}+\omega_{3, x}\right)\right]
\end{aligned}
$$

As $\delta u, \delta v$ are the variations of the independent quantities, then we obtain from variation of $L$ wrt $u, v$ (with account of (31)):

$$
\begin{gathered}
\omega_{1, x}+\omega_{3, y}=0 ; \omega_{2, y}+\omega_{3, x}=0 \quad \text { in } \Gamma \\
\omega_{1} \cos (x, n)+\omega_{3} \cos (y, n)=0 \quad ; \omega_{3} \cos (x, n)+\omega_{2} \cos (y, n)=0 \quad \text { at } C_{1} \\
\gamma_{1}=\gamma_{2}=0 \quad \text { at } C_{2}
\end{gathered}
$$

But (32)-(33) leads to

$$
\omega_{1}=\omega_{2}=\omega_{3}=0
$$

Hence, (30) is equal to zero.

Finally, omitting some simple transformations, we obtain:

$$
\begin{aligned}
& \delta L=\int_{\Gamma} d \Gamma\left\{\vec{N}^{(0) T}\left[\begin{array}{c}
-\alpha_{1, x}+\frac{\lambda w_{, x}^{2}}{2 W^{(0)}} \\
-\alpha_{2, y}+\frac{\lambda w_{, y}^{2}}{2 W^{(0)}} \\
-\alpha_{1, y}-\alpha_{2, x}+\frac{\lambda w_{, x} w_{, y}}{2 W^{(0)}}
\end{array}\right]\right\}+\delta_{D}\left[\frac{\widetilde{\Pi}}{W^{(0)}}\right]+ \\
& +\int_{C_{1}} d C \delta \vec{N}^{(0) T}\left[\begin{array}{c}
\beta_{1} l+\alpha_{1} l \\
\beta_{2} m+\alpha_{2} m \\
\beta_{1} m+\beta_{2} l+\alpha_{2} l+\alpha_{1} m
\end{array}\right]+\int_{C_{2}} d C \delta \vec{N}^{(0) T}\left[\begin{array}{c}
\alpha_{1} l \\
\alpha_{2} m \\
\alpha_{2} l+\alpha_{1} m
\end{array}\right]
\end{aligned}
$$

where $\delta \vec{N}$ contains the independent variations. It follows from (36) that

$$
\alpha_{1}=\alpha_{2}=0 \text { at } C_{2}
$$


and

$$
\alpha_{1}=\beta_{1} \quad ; \alpha_{2}=\beta_{2} \quad \text { at } C_{1}
$$

Also

$$
-\alpha_{1, x}+\frac{\lambda w_{, x}^{2}}{2 W^{(0)}}=0 \quad ;-\alpha_{2, y}+\frac{\lambda w_{, y}^{2}}{2 W^{(0)}}=0 \quad ;-\alpha_{1, y}-\alpha_{2, x}+\frac{\lambda w_{, x} w_{, y}}{2 W^{(0)}}=0 \quad \text { in } \Gamma
$$

The relations (37) and (39) lead to the determination of $\alpha_{1}, \alpha_{2}$.

The only item in (36) with the variation of the ply angles is

$$
\delta_{D}\left[\frac{\widetilde{\Pi}}{W^{(0)}}\right]=\sum_{i=1}^{K} \delta \theta_{i}\left\{-4 U_{3} h_{p l y} \sin 2\left(\theta_{i}-\psi\right)\left[\frac{U_{2}}{4 U_{3}}\left(k_{1}^{2}-k_{2}^{2}\right)+\left(k_{1}-k_{2}\right)^{2} \cos 2\left(\theta_{i}-\psi\right)\right] \frac{1}{6}\left(z_{i}^{3}-z_{i-1}^{3}\right)\right\}
$$

where $k_{1}, k_{2}, \psi, z_{i}, z_{i-1}$ are the largest and the lowest principal curvatures, the angle between the direction $k_{1}$ and the $\mathrm{X}$ axis, the top and the bottom $z$ coordinates of the $i$-th layer, $i=1, \ldots, K$. The relation (40) was obtained in more simplified case in (Selyugin, 2013) using the Section 2 background on composites.

The relation (40) means that the lay-up optimality conditions are

$$
\sin 2\left(\theta_{i}-\psi\right)\left[\frac{U_{2}}{4 U_{3}}\left(k_{1}^{2}-k_{2}^{2}\right)+\left(k_{1}-k_{2}\right)^{2} \cos 2\left(\theta_{i}-\psi\right)\right] \frac{1}{6}\left(z_{i}^{3}-z_{i-1}^{3}\right)=0, i=1, \ldots, K
$$

The conditions are identical with the conditions obtained without account of the in-plane force variation (Selyugin, 2013).

\section{DISCUSSION}

The above-obtained results clearly demonstrate that there is an influence of the $2 \mathrm{D}$ stress distribution on the buckling eigenvalue. The influence is due to the quantity $W^{(0)}$ in the denominator of (14). The quantity contains the in-plane forces. 


\section{CONCLUSIONS}

- The first order necessary local lay-up optimality conditions for the plate buckling level maximization are derived;

- The influence of the in-plane forces to the optimal buckling eigenvalue comes through the denominator quantity $W^{(0)}$ containing the forces;

- The derived optimality conditions may be used for obtaining the proper lay-up sensitivities

\section{ACKNOWLEDGEMENT}

The author is thankful to the colleagues for the fruitful discussions.

\section{REFERENCES}

Falzon BG, Aliabadi MH Buckling and postbuckling structures: experimental, analytical and numerical studies, Imperial College Press, 2008.

Ghiasi H, Pasini D, Lessard L 2009. Optimum Stacking Sequence Design of Composite Materials Part I: Constant Stiffness Design. Compos Struct 90: 1-11.

Ghiasi H, Fayazbakhsh K, Pasini D, Lessard L 2010. Optimum Stacking Sequence Design of Composite Materials Part II: Variable Stiffness Design. Compos Struct 93: 1-13

Gibson RF 1994. Principles of composite material mechanics. McGraw-Hill, Inc., 425 pp. 
Ijsselmuiden ST, Abdalla MM, Gürdal Z. 2010. Optimization of variable-stiffness panels fo Maximum buckling load using lamination parameters. AIAA J, 48: 134-143.

Kassapoglou C 2010. Design and Analysis of Composite Structures: With Applications to Aerospace Structures. Wiley.

Lekhnitzky SG 1956. Anisotropic plates. (translation from Russian), American Iron and Steel Institute, New York, 1956.

Ohsaki M, Ikeda K 2007. Stability and Optimization of Structures. Generalized Sensitivity Analysis. Springer

Reddy JN 2004. Mechanics of laminated composite plates and shells. Theory and analysis. $2^{\text {nd }}$ edition. CRC Press, 831 pp.

Selyugin S 2019a. Lay-up optimality conditions for stiffness maximization of anisotropic composite plates in postbuckling. https://doi.org/10.31224/osf.io/k2rwp

Selyugin S 2019b. A relation between lamination parameters of stiffest post-buckled composite plate. https://doi.org/10.31224/osf.io/ctzue

Selyugin S 2013. On Choice of Optimal Anisotropy of Composite Plates against Buckling, with Special Attention to Bending-twisting Coupling. Struct Multidisc Optim 48: 279-294 
Turvey GJ, Marshall IH (eds) 1995. Buckling and postbuckling of composite plates. Chapman and Hall.

Volmir AS 1967. Stability of Elastic Systems (in Russian), Moscow, Nauka Publ. English Translation: Foreign Technology Division, Air Force Systems Command. Wright-Patterson Air Force Base, Ohio, AD628508.

Washizu K 1982. Variational methods in elasticity and plasticity. Pergamon Press, Oxford. 